\title{
Digital Capacitance and Inductance Meter
}

\author{
M. Hruškovic, J. Hribik \\ Dept. of Radio Electronics, Faculty of Electrical Engineering and Information Technology, Slovak University of Technology, \\ Ilkovičova 3, 81219 Bratislava, Slovak Republic, e-mail: hruskovic@kre.elf.stuba.sk; jan.hribik@stuba.sk
}

\begin{abstract}
A microcomputer-controlled measuring instrument for capacitance and inductance measurement is described. It is based on an oscillator circuit with the oscillation frequency dependent on a measured element. An analysis of the oscillator used is also given. Equations for the oscillation frequency and its deviation from the resonance frequency of a frequency controlling resonance circuit are derived. The measured results can be transferred into a personal computer (PC) which can process and display these results and control the instrument via RS-232 serial interface.
\end{abstract}

Keywords: capacitance and inductance measurement, microcomputer control

\section{INTRODUCTION}

$\mathrm{E}$ LECTRONIC METHODS of capacitance and inductance measurement are based on different principles [1]-[4]. They include bridge methods, vector impedance methods, resonance methods, digital RLC methods, voltage and current measurement methods, and phase shift measurement methods. All of these methods have some advantages but also suffer from some drawbacks.

The most precise are bridge methods but the construction and measurement by such instruments are complicated. Digital RLC methods use feedback amplifiers and low measurement frequencies [2], [5]. The measurement circuit in these methods is simple but the accuracy is relatively low. Resonance methods use the known dependence of the resonance frequency on the values of an inductance and capacitance elements of a series or parallel resonance circuit. For the finding of resonance frequency, they are inaccurate.. A modified version of the resonance methods is based on the measurement of the frequency of an oscillator with the measured element connected into a frequency controlling resonance circuit. Digital frequency measurement is accurate and yields the desired digital output of the instrument.

\section{PRINCIPLE OF THE PROPOSED METHOD}

The method used in the designed instrument is based on the oscillator, the frequency of which depends on the measured value of an inductance or capacitance. A simplified circuit diagram of the oscillator used is in Fig.1 [6]. A resistor $R_{L P}$ represents the losses in the coil supposing that the capacitor has negligible losses. The condition for the oscillations (Barkhausen criterion), [7], is given by the expression

$$
\boldsymbol{\beta}(j \omega) \mathbf{A}(j \omega)=1
$$

where $\boldsymbol{\beta}(j \omega)=\beta(\omega) \exp \left[j \varphi_{F B}(\omega)\right]$ is the feedback circuit transfer function, $\mathbf{A}(j \omega)=A(\omega) \exp \left[j \varphi_{A}(\omega)\right]$ is the amplifier transfer function, and $\omega$ is the complex frequency. From (1), the amplitude and the phase conditions yield these expressions:

$$
\begin{aligned}
& \beta(\omega) A(\omega)=1 \\
& \varphi_{F B}(\omega)+\varphi_{A}(\omega)=0
\end{aligned}
$$

From Fig.1, the feedback transfer function can be written in the form

$$
\boldsymbol{\beta}(j \omega)=\frac{\omega^{2} L^{2} R_{L P}\left(R_{L P}+R_{F B}\right)+j \omega L R_{L P}^{2} R_{F B}\left(1-\omega^{2} L C\right)}{R_{L P}^{2} R_{F B}^{2}\left(1-\omega^{2} L C\right)^{2}+\omega^{2} L^{2}\left(R_{L P}+R_{F B}\right)^{2}}
$$

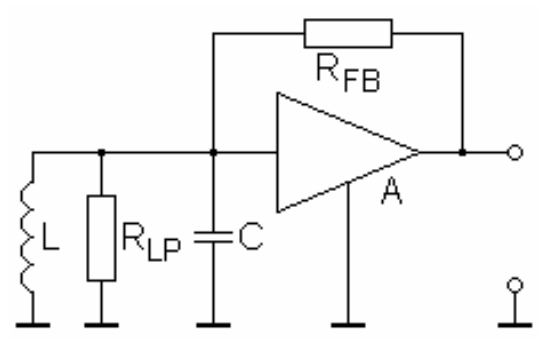

Fig.1 Simplified circuit diagram of the oscillator

Notice that at the resonance frequency, $\omega_{r},(4)$ simplifies to the expression

$$
\beta(\omega)=\frac{R_{L P}}{R_{L P}+R_{F B}}=\frac{Q \omega_{r} L}{Q \omega_{r} L+R_{F B}}
$$


and from the amplitude condition (2)

$$
A(\omega)=1+\frac{R_{F B}}{Q \omega_{r} L}
$$

In these equations the resonance frequency, $\omega_{r}$, and the Q-factor of the resonance circuit

$$
\begin{aligned}
& \omega_{r}=\frac{1}{\sqrt{L C}} \\
& Q=\frac{R_{L P}}{\omega_{r} L}
\end{aligned}
$$

were introduced.

The phase characteristic of the feedback circuit can now be expressed from (4). Near the resonance frequency this expression is

$$
\varphi_{F B}(\omega)=\tan ^{-1} \frac{Q R_{F B}\left(1-\omega^{2} L C\right)}{\omega L Q+R_{F B}}
$$

and it can be seen that $\varphi_{F B}(\omega)$ approaches zero. In this case it is possible to express the oscillation frequency in the form

$$
\omega^{2}=\frac{1}{L C}\left(1-\frac{\varphi}{Q} \frac{A(\omega)}{A(\omega)-1}\right)
$$

where $\varphi_{A}(\omega)=-\varphi$ is the amplifier phase shift at the oscillation frequency. The relative deviation of the $\omega^{2}$ from the resonance frequency $\omega_{r}^{2}$ is

$$
\delta\left(\omega^{2}\right)=-\frac{\varphi}{Q} \frac{A(\omega)}{A(\omega)-1}
$$

\section{DESIGNED INSTRUMENT DESCRIPTION}

A block diagram of the designed instrument for inductance and capacitance measurement is in Fig.2 [6]. A measured value-to-digital-value converter contains a measuring oscillator, an amplitude control circuit, input protection circuits and a harmonic-to-rectangular signal shaper, Fig.3. The measuring oscillator is composed of an amplifier, a resonance circuit, reference elements and a relay switch controlled from a microcomputer part. The rectangular shaped signal is lead to the microcomputer part which measures the oscillation frequency, calculates the measured value and displays the result on the LED display. The microcomputer part also operates a control keyboard and a serial interface (RS232) to a personal computer (PC) and controls the operation of the instrument.

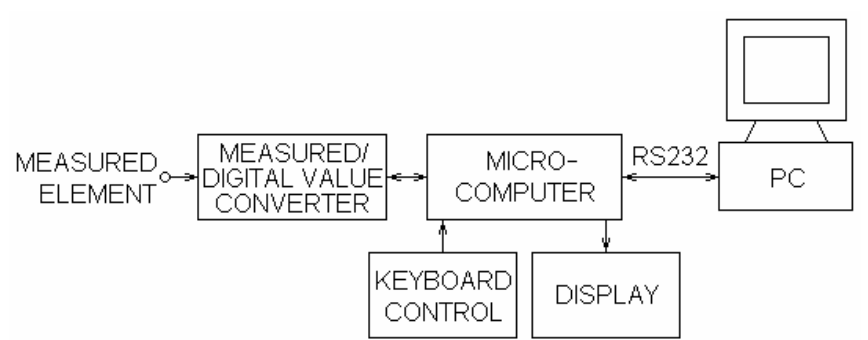

Fig.2 Block diagram of the designed measuring instrument

The measuring oscillator is of the type shown in Fig.1. For capacitance measurement, a reference inductor is connected in parallel into the resonance circuit by a relay switch, Fig.3. The reference inductor is realized by a capacitive loaded gyrator with two operational amplifiers (OA). Its inductance is $78 \mathrm{mH}$ and is linear up to the current $11 \mathrm{~mA}$. It is enough to measure the capacitances up to $10 \mu \mathrm{F}$. Such high inductance cannot be realized by a coil with a core because of its nonlinearity and temperature dependence. A coil without a core has high number of turns with high DC resistance and high selfcapacitance.

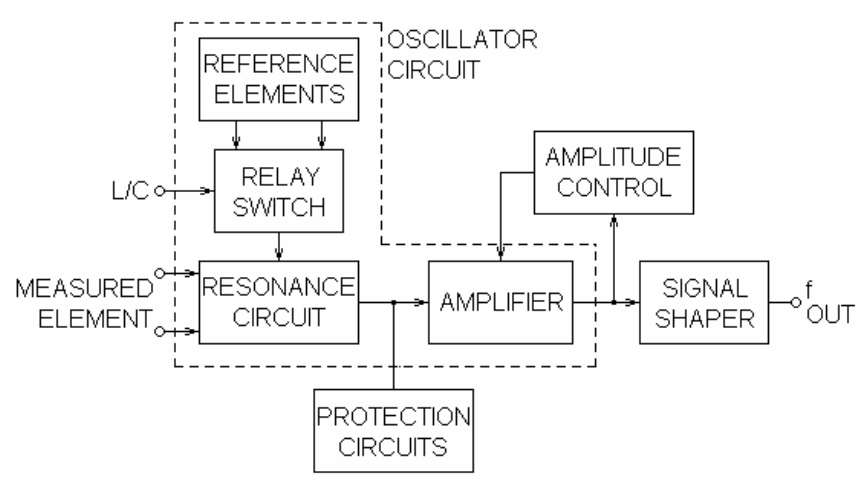

Fig.3 Block diagram of the measured value-to-digital value converter

For inductance measurement, a reference capacitor is connected in parallel into the resonance circuit by a relay switch. Its capacitance is selected to be $20 \mathrm{nF}$ and is composed of two $10 \mathrm{nF}$ capacitors. This choice was made to get approximately the same range of the measured capacitive and inductive reactances.

The amplitude of the oscillations is partially controlled by anti-parallel connection of two diodes in a negative feedback which is used to set the amplifier gain. Much more effective amplitude control is realized by a control of the feedback resistor. For these purposes the feedback resistor is a photo resistor and is controlled by the rectified and amplified oscillator output signal through an LED. 
To prevent the damage of the oscillator, e. g. when connecting a charged measured capacitor, the high-speed inversely biased diodes with low capacitance are used. Another protection is a connection of resistors into the inputs of the OA with a diode protection inside the OA.

The oscillator output signal is shaped to a rectangular form by a comparator with hysteresis. In this way, the TTL/CMOS compatible rectangular signal is obtained for the microcontroller to measure its frequency.

\section{MICROCOMPUTER PART OF THE INSTRUMENT}

The microcomputer part of the instrument must measure the frequency or period of the oscillator signal, calculate the measured value, display the measurement results, control the whole instrument, communicate with a PC and perform some other auxiliary functions. All these requirements can be satisfied by a designed microcomputer circuit and peripherals shown in Fig.4. A core of this part is a microcontroller. Its choice came out of the following requirements: minimum of two 16-bit timer/counters to enable to measure the oscillator frequency in the whole range with sufficient accuracy, sufficient number of I/O ports, supporting of a serial interface, sufficient computing power, sufficient capacity of program and data memory (possibly EEPROM type to enable to store calibration constants).

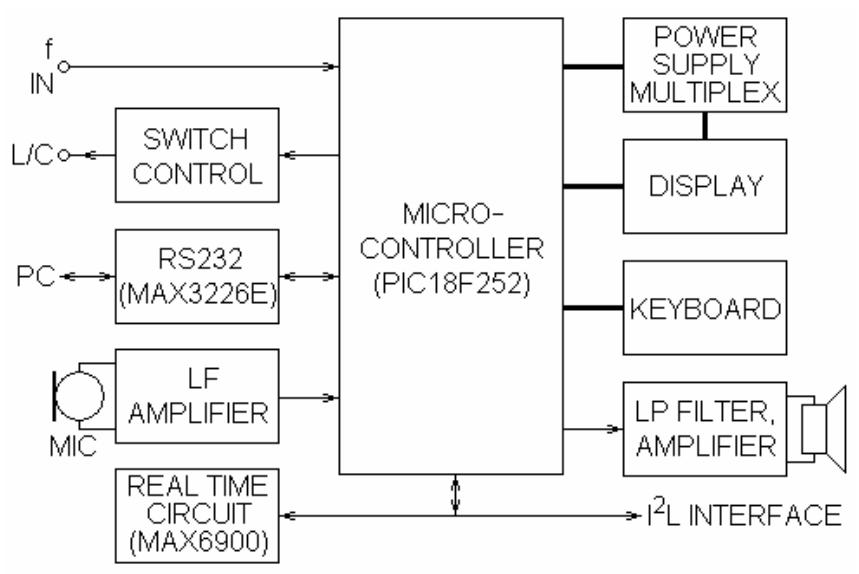

Fig.4 Block diagram of the microcomputer part of the instrument

The microcontroller PIC18F252 has RISC CPU with maximum clock frequency $40 \mathrm{MHz}$ and maximum speed of operation up to 10 MIPS. This clock frequency can be obtained from $10 \mathrm{MHz}$ crystal oscillator by means of a built-in PLL. This possibility is used in the designed instrument. The microcontroller contains three memories: 1.5 Kbytes data RAM, 32 Kbytes FLASH program memory and 256 bytes data EEPROM. The FLASH program memory is readable, writable and erasable during normal operation. In the designed instrument it is possible to change the program in this memory through a serial port and to write samples of a voice control of the instrument into it. The data EEPROM is used to store calibration constants.

From the frequency or period measurement point of view it is important that the microcontroller PIC18F252 contains one 8-bit and three 16-bit timers/counters with programmable prescalers. Other important peripheral features of the microcontroller are two CCP (Capture/Compare/PWM) modules, a synchronous serial port module (two modes of operation: 3-wire SPI, $\mathrm{I}^{2} \mathrm{C}$ ) and an addressable asynchronous serial port module (supports RS-485 and RS-232). Analogue features of the microcontroller are a 10-bit A/D converter module with 5-channel multiplexed input, programmable low voltage detection and other special features such as watchdog timer with its own on-chip RC oscillator, power saving SLEEP mode, selectable oscillator options, etc.

A LED display of the instrument is multiplexed, which means that the power supply voltage of the common anodes is switched by transistors controlled from port A every $10 \mathrm{~ms}$. The cathodes are controlled from port $\mathrm{B}$ of the microcontroller. The keyboard microswitches are sensed through port $\mathrm{B}$ and port $\mathrm{RC} 5$ of the microcontroller. The relay switch, which selects $\mathrm{L}$ or $\mathrm{C}$ measurement, is controlled from port $\mathrm{RC} 1$. The oscillator signal is connected to the timer 1 input TMR1.

A TTL/RS-232 level converter MAX3226E is connected to pins RX and TX (serial port). This circuit supports maximum transfer speed $250 \mathrm{Kbits} / \mathrm{s}$ and has an electrostatic discharge protection. To minimize the interference and power consumption, this circuit is active only if it is connected to the active RS-232 interface or if it transmits data. In other case it is in SLEEP mode.

The instrument also makes use of other supplementary functions. One of them is $I^{2} \mathrm{~L}$ interface. The data and clock lines are electrostatic discharge protected and a $+5 \mathrm{~V}$ power supply line with current limit and overvoltage protection is added. A temperature sensor DS1631A and a real time circuit MAX6900 are also connected to this $\mathrm{I}^{2} \mathrm{~L}$ line. The A/D converter is used to digitize a voice control input signal from a microphone, which is filtered and amplified before conversion. PWM module (pulse frequency $156 \mathrm{kHz}, 8$-bit resolution) is used to create a voice output from the input sampled signal with 8 -bit resolution and $8 \mathrm{kHz}$ sampling frequency. A low-pass reconstruction filter at its output has the limiting frequency $4 \mathrm{kHz}$ and the signal is next amplified and connected to a loudspeaker. Thus, the instrument can announce some events such as range switching, etc.

\section{EXPERIMENTAL RESULTS}

The realized instrument is able to measure capacitances from $1 \mathrm{pF}$ up to $10 \mu \mathrm{F}$ and inductances from $1 \mu \mathrm{H}$ up to $15 \mathrm{H}$ (higher values have not been tested). The deviations of the measured capacitances and inductances from those calculated from the Thomson equation are less than $1 \%$. Because of low dispersion of the measured values it is possible to use software 
correction in the microcontroller. The deviation of the oscillator frequency is independent on the Q-factor and is very low for the Q-factors of the resonance circuit higher than 2. The error of measurement caused by temperature changes is $0.06 \% /{ }^{\circ} \mathrm{C}$. The overall accuracy of the instrument after calibration is $0.3 \%+1 \mathrm{pF}$ or $0.3 \%+1 \mu \mathrm{H}$.

\section{CONCLUSION}

A microcomputer-controlled measuring instrument for capacitance and inductance measurement based on the oscillator circuit with the oscillation frequency dependent on the measured element was designed. The analysis of the oscillator used yields the equations for the oscillation frequency and its deviation from the resonance frequency of a frequency controlling resonance circuit. The analogue and digital part of the instrument are described following block diagrams in Fig.2, Fig.3 and Fig.4. The basic circuit of the analogue part is the measuring oscillator while the core of the digital part is a microcontroller. The realized instrument is able to measure capacitances from $1 \mathrm{pF}$ up to $10 \mu \mathrm{F}$ and inductances from $1 \mu \mathrm{H}$ up to $15 \mathrm{H}$ (higher values have not been tested). The overall accuracy of the instrument after calibration is $0.3 \%+1 \mathrm{pF}$ or $0.3 \%+1 \mu \mathrm{H}$ in the whole measuring range. The measured results can be transferred into a personal computer (PC) which can process and display these results and control the instrument via RS-232 serial interface. The instrument equipment includes temperature measurement, real time and date, serial interface $\mathrm{I}^{2} \mathrm{~L}$, voice control input and voice output.

\section{ACKNOWLEDGMENT}

This work was partially supported by the Slovak grant agency, GAT, under the grant VEGA No. 2/0107/08 and the project No. AV 4/0012/07.

\section{REFERENCES}

[1] Helfrick, A.D., Cooper, W.D. (1990). Modern Electronic Instrumentation and Measurement Techniques. Englewood Cliffs, USA: Prentice-Hall.

[2] Sedláček, M., Haasz, V. (2000). Electrical Measurements and Instrumentation. (2nd ed.). Prague, Czech Republic: Vydavatelství ČVUT.

[3] Webster, J. (ed.) (1999). Wiley Encyclopedia of Electrical and Electronics Engineering Online. Instrumentation and Measurement. New York, USA: John Wiley \& Sons. (http://mrw.interscience.wiley.com/emrw/9780471346081 /home/)

[4] Hribik, J. (2002). Electronic Measurement. Bratislava, Slovak Republic: Vydavatel'stvo STU. (In Slovak)

[5] Lentka, G., Hoja, J. (2004). The influence of sampling parameters on accuracy of capacitance measurement in the method based on DSP. In $13^{\text {th }}$ International Symposium on Measurements for Research and Industry Applications and $9^{\text {th }}$ European Workshop on ADC Modelling and Testing, Volume 1, 29 September-1 October 2004 (pp. 294-297). Athens, Greece: NTUA and IMEKO TC-4.

[6] Bednáŕ, V., Hruškovic, M. (2004). Capacitance and Inductance Meter. Bratislava, Slovak Republic: FEI SUT. (In Slovak)

[7] Sentz, R.E., Bartkowiak, R.A. (1968). Feedback Amplifiers and Oscillators. New York, USA: Holt, Rinehart and Winston. 